\title{
Cognitive Functioning Differences Between Physically Active and Sedentary Older Adults
}

\author{
Ben Younan* \\ Bond University, Gold Coast, Australia
}

\begin{abstract}
Older adults engaging in regular physical activity can help create a buffer toward cognitive decline. The main aim of this study was to assess the effects of physical activity and cognitive functioning on a sample of young-old and old-old physically active adults and young-old and old-old sedentary adults. Cognitive functioning was examined using the digit span test, Wisconsin card sorting task. The National Task Group-Early Detection Screen for Dementia measure was used explore the relationship between scores and physical activity and sedentary adults. Findings from the study showed partial support for physical activity has a positive relationship with cognitive functioning. The results found Young-old adults did better on the DST than all other groups regardless of being sedentary or physically active. Young-old physically active adults, however, did better on the DST latency measure than sedentary adults while old-old physically active adults did worse on the DST latency measure compared with old-old sedentary adults. The results also found that Physically active adults had a better score on the NTG-EDSD measure compared to the sedentary adults. Findings showed no significant differences for the WCST. Future research exploring the relationship between physical activity and cognitive functioning should do so by having more control over the extraneous variables.
\end{abstract}

Keywords: Alzheimer's disease, cognitive decline, old-old, older adults, physically active, young-old

\section{INTRODUCTION}

Older age is accompanied by many changes within the human body. One of the most recognized changes includes a decline in cognitive functioning [1]. Cognitive decline is a universal age-related problem that occurs from middle age onwards, however, in some cases, can occur even earlier. Age-related cognitive impairments are usually gradual and produce declines in processing speed, reasoning skills, memory, and executive functions [2,3]. A common result of cognitive decline is dementia [4]. Dementia is characterized by a chronic progressive decline of the brain that affects memory, thinking, orientation, comprehension, calculation, learning capacity, language, and

\footnotetext{
*Correspondence to: Ben Younan, Bond University, Gold Coast, Australia. E-mail: younanben@gmail.com.
}

judgment $[2,5,6]$. These impairments in cognitive function can also be accompanied by deterioration in emotional control, social behavior, or motivation [6]. The onset of dementia also limits the ability of people who suffer from the disease to perform at least one basic task such as everyday self-care, mobility or basic communication tasks [5]. Dementia negatively impacts cognition whereby individuals have a reduced ability to carry out everyday tasks.

The literature has identified the main factors associated with resilience towards cognitive decline, these include physical activity, education, cognitive training, healthy diet, social engagement, and an overall good mental health [7, 8]. Magnetic resonance imaging (MRI) studies have found that physical activity increases both grey and white brain volume in the frontal, occipital, entorhinal, and hippocampal regions [9-11]. Older adults who engage in regular 
physical activity also report better cognitive functioning than sedentary adults [12]. Research has also found that executive functioning can also be improved in adults diagnosed with mild to moderate Alzheimer's disease through regular physical activity [13].

The cognitive functioning of people with dementia changes within a person's lifespan based on influences that can encourage positive or negative neuroplasticity [14]. Neuroplasticity is the brains natural ability to increase or decrease the amount of neural connections between different brain regions based on intrinsic and extrinsic influences [15]. Positive neuroplasticity is based on the physiological response of the brain to enhance or repair neural connections [14]. This is a radical and recent change from the dominant model of the last century, which largely ruled out neurogenesis in adults [15].

Positive neuroplasticity is promoted by regular physical activity, education, social interaction, intellectual pursuits, and cognitive remediation. Negative neuroplasticity is also based on the physiological ability of the brain, though it is based on weakening dendritic connections, which produce damaging morphological changes and the tendency for connections to degrade over time [16]. The factors that encourage negative neuroplasticity comprise of poor health, poor sleep hygiene, poor nutrition, substance abuse, and depression and anxiety. Fritsch et al. [8] conducted a study to look at whether cognitive reserve is static or dynamic and could be increased over a person's lifespan. The researchers also looked at when the optimal time for improvements were best to try and improve cognitive reserve. The results of the study showed that cognitive reserve is dynamic and can be increased over the lifespan with the optimal time being within the first two decades of life.

Physical activity may also facilitate protection against Alzheimer's by helping develop healthy synapses or neurons, which increases the amount of remaining available neurons in the brain, so when there is a decrease on synapses in the brain due to aging there is still enough to compensate [17]. Exercise activates molecular and cellular cascades that maintain brain plasticity [15]. It helps produce changes in genes that are associated with plasticity and promotes vascularization, neurogenesis, functional changes in neuronal structure and neuronal resistance to injury [16]. These affect areas within the hippocampus, and brain areas associated with learning and memory [18]. Exercise can help facilitate cellular growth, which slows down the cognitive effects of Alzheimer's.
The examination of physical activity and cognitive functioning among older adults typically separates young-old (55-70 years) and old-old (71+- years old) [19-21]. This is important because it will help with understanding whether physical activity can aid in preventing or slowing down any cognitive functioning. The main aim of the current study was to compare the cognitive functioning (memory, executive functions) between young-old and old-old physically active and sedentary subjects. The current study also explored the relationship between scores on the National Task Group-Early Detection Screen for Dementia (NTG-EDSD) among physically active and sedentary adults.

Previous studies have indicated that instruments like the Mini Mental State Exam (MMSE) and Montreal Cognitive Assessment (MOCA) to not be sensitive measures, nor specific measures for detecting memory deficits in individuals with MCI or those in early stages of dementia [22-24]. One way to address the deficits of the MMSE is to use a more sensitive measure which is why the current study looked at utilizing the NTG-EDSD [25]. The NTG-EDSD is a new measure for adults with both intellectual and developmental disability that is suggestive of MCI or dementia based on changes in thinking, behavior and adaptive skills [25]. It is recommended for use with people over the age of 40 [25]. The current study used the NTG-EDSD as a dependent variable to explore whether there would be differences in scores among sedentary and physically activity adults. Exploring the NTG-EDSD as a dependent variable was what made this study unique.

To examine cognitive functioning in a sample of older adults the current study used two computerized neuropsychological measures. This included the Wisconsin card-sorting task (WCST) and digit span test (DST). The WCST is among the most commonly used measures for executive functioning while DST is among the most commonly used measures for memory [26]. The WCST assesses executive and frontal lobe functioning through an individual's ability to form abstract concepts, the ability to shift and maintain attention, and to utilize feedback [27, 28]. The DST is another commonly used measure of cognitive functioning in older adults [29-35]. Older adults who engage in physical activity have been shown to have higher levels of cognitive functioning compared to sedentary older adults [36, 37]. It was hypothesized that physically active adults would have better cognitive functioning than sedentary adults. It was also hypothesized that physically active adults would 
score significantly lower on the NTG-EDSD than sedentary adults.

\section{METHODS}

\section{Participants}

The sample was comprised of 55 male and female participants. Participants were placed into four groups based on age and whether they participated in any type of physical activity. The ages of participants ranged from 45 to 94 years $(M=63.29$, $\mathrm{SD}=13.77$ ). A young-old physically active group comprised of 25 members aged between 45 and 67 years $(\mathrm{M}=55.6, \mathrm{SD}=6.31)$; a young-old sedentary group comprised of 13 members aged between 45 and 67 years $(\mathrm{M}=53.53, \mathrm{SD}=4.78)$; an old-old physically active group comprised of 6 members aged between 72 and 94 years $(\mathrm{M}=79.16, \mathrm{SD}=5.91)$; and an old-old sedentary group comprised of 11 members aged between 72 and 94 years $(M=83.00, S D=6.92)$. Categorizing the participants into the two groups (young-old and old-old) was based on previous literature that used similar age groups $[19,21]$.

Participants were recruited via the student research participation pool at Bond University, local gyms, senior citizen organizations, and residential housing within Gold Coast, Queensland. The study was conducted in accordance with the ethics approved by the Bond University Human Research ethics committee (BUHREC). In order to avoid causing unfounded concerns about performance based on measures, participants were not provided individual results.

In order to be included in the physically active groups, participants had to engage in exercise that included aerobic or muscle strengthening activity that was planned, structured and required repetitive movement that allowed for improved or maintained level of physical fitness [38]. The sedentary group consisted of participants that did not engage in any regular physical activity. Participants data were excluded from the study if they did not speak fluent English, had impairments that affected cognitive functioning (MCI, dementia, depression, anxiety), had taken any medication that affected cognitive function, or taken illicit drugs within the last twelve hours of testing. In addition, participants who had consumed two or more standard alcoholic beverages within the last two hours prior to testing were also excluded.

Furthermore, participants were excluded from the study if they scored in the severe range for anxiety and depression using the Depression Anxiety and Depression Scale (DASS). To eliminate ethical dilemmas within the study, all recruited participants results on the DASS and the inclusion criteria were assessed after participants had completed the study and the researcher had left the testing premises. This was done as to not allow for any confrontation between participants and experimenter if they did not meet the criteria to have their data be included in the study. Participants in the study were informed of the ethical approval for the study, given by BUHREC and were asked to read and sign the informed consent giving the researchers permission to use their data in the study. All participants were reassured that their participation in the study would be confidential.

\section{Materials}

The materials used in the study included two paper and pen instruments and two computerized neuropsychological tests. The paper and pen instruments included the DASS and the NTG-EDSD. The DASS was included as inclusion criteria. The two computerized tests included the WCST and the DST. The two neuropsychological tests were designed to assess areas of attention, memory and executive functioning and the NTG-EDSD was used to examine differences between age and physically active and sedentary adults. The DST (span, latency), WCST (correct responses, total errors, perseverative responses, perseverative errors, and non-perseverative errors) and the NTG-EDSD (total score) were the dependent variables and the independent variables included a physically active group and age group.

\section{DST}

The DST is common among most research on cognitive functioning and it is used to test shortterm memory and immediate recall abilities, which involves visual attention, and the ability to remember a list of numbers [29, 32]. Research has found differences between different ages, particularly indicating declines within older adults [39]. GrÉGoire and Van Der Linden [40] Looked at the differences between forward and back digit span and found no differences although did find that after the age of 65 performances on both tests found significant declines in performance. The test was a simple forward digit span test and was presented to participants electronically. The internal consistency for the digit span test is reported as being above 0.90 , which is very good and test re-test reliability is also seen as being high, between 0.80 to 0.89 [27]. 
To assess accurate memory recall participants were presented with a list of numbers on the computer screen for a period of $2000 \mathrm{~ms}$ followed by a brief black blank screen for a further period of $500 \mathrm{~ms} \mathrm{sec-}$ onds. Participants were then instructed by a prompt on the computers screen to type in the digits that had appeared on the previous screen in the correct order using the external number pad beside the computer [41]. Cognitive processing speed was recorded from the first press of the keypad until completion of the span.

The administration of the DST using a computerized version allowed for clarity in delivering digit list and eliminated any mistakes in adjusting the length of the digit list. The list of digits was increased by increments of one and every time the participants would recall the digit correctly and decreased by one every time the participant recalled incorrectly. The test started with a minimum of three digits and increased towards a maximum upper limit of thirty-three digits. The test would run for thirty trials or when the participant plateaued. This allowed for shorter testing time and optimized the sampling performance by measuring participant's performance that was closest to their memory capacities [42].

\section{WCST}

The WCST measures executive functioning through cognitive flexibility (the ability to establish a set of options for finding a solution towards a perceived problem), set shifting ability (the ability to adjust when there is a shift in the rule and the ability to maintain a set rule when there is no shift in the rule), working memory and processing speed [42]. It requires strategic planning, organized searching, the ability to use environmental feedback to shift cognitive set, goal-oriented behavior, and the ability to modulate impulsive reasoning [27]. It was administered using the psychology experiment building language (PEBL) test battery version 0.13 [41].

The rules of the WCST were to sort a single card into a set pile of four individual cards. The sorting of the cards is based on several options of the rule (color, form, and number), which change regularly during the test and the participant had to adjust to each change accordingly. The participants were not given any instructions on how to sort the cards but had to decide what the card sorting principles were through the response that was given from the program after each trial of the card sorting. The response that was given was a simple 'correct' or 'incorrect' based on the move that the participant takes [43]. The participants were only given feedback on whether they were correct or incorrect once they decided which pile the card they were given belonged to. Each participant completed a total 128 trials. Scoring was based on the number of correct responses, the amount of total errors, perseverative responses, perseverate errors, non-perseverate errors, unique errors, and the failure to maintain set.

\section{DASS}

The DASS was used as an inclusion criterion. For this study, the DASS-21 item scale was used [44]. Participants responses varied based on four options; "Did not apply to me at all", "Applied to me to some degree, or some of the time", "Applied to me to a considerable degree, or a good part of time" and "Applied to me very much, or most of the time". The DASS-21 includes items that assess depression (e.g., "I felt that life was meaningless"), anxiety (e.g., "I felt scared without any good reason") and stress (e.g., "I felt that I was rather touchy"). Participants who scored high on the depression subscale were excluded from the study. The depression subscale included seven items. Internal consistency for the DASS has been found to be high in multiple studies [45, 46]. Henry and Crawford [47] reported that the internal consistency of the total scale for the DASS to be high with a Cronbach's Alpha of 0.96 and a Cronbach's Alpha of 0.93 for the depression subscale. Retest reliability has shown stable coefficients on responses over a twoweek period for depression $r=0.71(p<0.001)$ [48]. Convergent validity between the depression subscale of the DASS and the Beck Depression Inventory has also been established $r=0.74$ [44]

\section{$N T G-E D S D$}

No set scoring system had been previously developed for the NTG-EDSD. Therefore, this study had participants complete the form and for every ticked response on each measure a point was given. The measure was originally designed for family and caregivers of older adults to note functional decline and health problems [25]. Within the present study all items on this measure were re-worded in order to be self-reported by each participant. The estimated time for completion of this form was between 15 to 60 minutes [25]. The NTG-EDSD included items that assessed activities of daily living (e.g., 'need help with washing and/or bathing'), language and communication (e.g., 'I do not follow simple instructions'), sleep-wake change patterns (e.g., 'I wander at night'), ambulation (e.g., 'I fall'), memory (e.g., 'I lose or 
misplace objects'), behavior and affect ('I withdraw from people'), self-reported problems (e.g., 'I hear things'), notable significant changes observed by others (e.g., 'In gait') and chronic health conditions (e.g., 'Arthritis').

\section{Procedure}

For the purpose of the current study all tests were completed either at the participants' homes or in a quiet room at Bond University. If there was a window in the room the participant's back was faced against the window to allow for minimal distractions. Participants were first provided with an explanatory statement. They were then asked to read and sign the informed consent form. They were then asked to complete the DASS and NTG-EDSD to the best of their ability. In order to create internal validity and minimize any researcher bias counterbalancing of each measurement was conducted between participants.

Participants were given brief resting time between the three measures to reduce fatigue and carryover effects. To reduce the potential for experimental bias and to ensure all examinees received standard format, the instructions for the computerized tests were delivered on the computer's screen and communicated verbally. The instructions for the pen-and-paper tests were read to the examinee according to the test publisher's format.

Once all testing was completed, participants were thanked for their time and reassured that their participation would remain anonymous and their results confidential. The forms detailing each participant's demographic information and their individual scores on the DASS were looked over to see if the requirements were met for each participants data to be included in the study. Those that did not meet the criteria had their information taken out of data sample.

\section{RESULTS}

A one way between-groups multivariate analysis of variance (MANOVA) was performed to investigate age and exercise on cognitive functioning. A separate MANOVA was carried out for DST (span, latency), and the WCST (correct responses, total errors, perseverative responses, nonperseverative errors). A between subject's ANOVA was performed on the total score of the NTG-EDSD. Three dependent variables were used: DST (span, latency), WCST (correct responses, total errors,
Table 1

Mean span and latency (ms) by age group

\begin{tabular}{lccccccc}
\hline Digit Span Test & \multicolumn{3}{c}{ Young-Old } & & \multicolumn{3}{c}{ Old-Old } \\
\cline { 2 - 4 } \cline { 6 - 7 } & $M$ & SE & $95 \%$ CI & & $M$ & SE & $95 \%$ CI \\
\hline Span & 6.6 & 0.20 & $(6.2,7.0)$ & & 5.5 & 0.29 & $(4.9,6.1)$ \\
Latency & 6920 & 111 & $(6696,7144)$ & & 7617 & 161 & $(7292,7942)$ \\
\hline
\end{tabular}

perseverative responses, non-perseverative errors) and the NTG-EDSD, all controlling for education. The independent variable was age and exercise. Preliminary assumption testing was conducted to check for normality, linearity, univariate and multivariate outliers, homogeneity of variance-covariance matrices, and multicollinearity, with no serious violations noted. Wilks' Lambda was used to assess significance at the $p=0.05$ level.

\section{Digit span test}

The results for the DST showed a significant main effect for age $(F(2,40)=10.49, p<0.04$; Wilks' Lambda $=0.65 ; \eta^{2}=0.34$, power $=0.98$ ). When considering the results of the dependent variables separately the only difference to reach statistical significance using a Bonferroni adjusted alpha level of 0.26 , was latency: $(F(3,42)=12.72, p<0.00$; $\eta^{2}=0.23$, power $=0.93$ ). Table 1 displays mean span and latency scores for the age groups.

Multivariate tests also found significant effects for the interaction between age categories and physical activity $(F(2,40)=3.42, p=0.04$; Wilks' Lambda $=0.85 ; \eta^{2}=0.14$, power $\left.=0.61\right)$. The tests of between subjects showed a significant interaction between age categories and physical activity specifically for latency, $\left(F(3,42)=4.41, p=0.04, \eta^{2}=0.09\right.$, power $=0.53$ ). The results shown in Table 2 indicate that the standard error was higher in the old-old group for latency, indicating a larger deviation from the mean compared to the results of the young-old sample. The confidence intervals showed that for latency there was no overlap between young-old and oldold, indication of a significant difference between groups. The results for latency showed a small standard error, indicating only a small deviation from the mean. The confidence intervals indicate an overlap between physical activity and age groups, which is an indication that there was no significant difference between groups among latency although significant.

The results for the DST showed no significant main effect for physical activity $(F(2,40)=0.59, p=0.94$; Wilks' Lambda $=0.99$ ). Although non-significant, the standard error was quite small and the 95\% 
Table 2

Mean latency to complete span response by age and physical activity groups

\begin{tabular}{lccccccc}
\hline & \multicolumn{3}{c}{ Young-Old } & & \multicolumn{3}{c}{ Old-Old } \\
\cline { 2 - 4 } \cline { 6 - 8 } & $M$ & SE & $95 \%$ CI & & $M$ & SE & $95 \%$ CI \\
\hline Active & 6746 & 131 & $(6481,7812)$ & & 7854 & 245 & $(7360,8348)$ \\
Sedentary & 7094 & 181 & $(6728,7460)$ & & 7380 & 214 & $(6949,7812)$ \\
\hline
\end{tabular}

confidence interval had an overlap between groups indicating no difference between the groups.

\section{WCST}

The results for the WCST showed no significant main effect for age categories $(F(4,28)=0.37$, $p=0.82$; Wilks' Lambda $=0.90)$; physical activity $(F(4,28)=0.44, p=0.77$; Wilks' Lambda $=0.91)$. There was also no significant main effect between the age categories with physical activity $(F(4,28)=1.14$, $p=0.35$; Wilks' Lambda $=0.99$ ).

\section{$N T G-E D S D$}

The results for the NTG-EDSD were assessed at the $p<0.05$ level. The results indicated there was a significant main effect for physical activity $(F(1$, $49)=11.62, p=0.001, \eta^{2}=0.01$, power $\left.=0.91\right)$. The confidence intervals showed that for the physically activity group there was no overlap between active and sedentary physically active groups, which indicates there was a difference in scores between active and sedentary individuals. Table 3 shows the main scores for the NTG-EDSD with main effect of physical activity.

The results indicated that there was no significant main effect for age groups $(F(1,49)=0.78, p=0.38)$. The standard error scores were low indicating good reliability although there was an overlap between the confidence intervals. There was also no significant main effect for the interaction between age and physical activity groups $(F(1,49)=0.63, p=0.42)$.

Table 3

Mean scores for NTG-EDSD with main effect of physical activity

\begin{tabular}{lcccc}
\hline & Mean & Std. Error & \multicolumn{2}{c}{ 95\% Confidence Interval } \\
\cline { 3 - 5 } & & & Lower Bound & Upper Bound \\
\hline Sedentary & 24.6 & 2.4 & 19.7 & 29.5 \\
Active & 11.2 & 3.1 & 4.9 & 17.4 \\
\hline
\end{tabular}

\section{DISCUSSION}

The current study was conducted to further investigate a widely held view in the literature that physical activity provides protective functioning against cognitive decline in older adults [12, 13]. Cognitive functioning levels among older adults were assessed using the DST and WCST. The study also explored the relationship between scores on the NTG-EDSD with physically active and sedentary adults. The NTG-EDSD is a new measure of early screens for dementia among older adults with both intellectual and developmental disability that is suggestive of MCI or dementia based on changes in thinking, behavior, and adaptive skills [25]. As previous research indicates that physical activity could act as a preventative measure for cognitive decline the study hypothesized that physically active adults would have better cognitive functioning than sedentary adults. It was also hypothesized that physically active adults would score significantly lower on the NTG-EDSD than sedentary adults.

The results showed that young-old adults were able to recall larger digit spans and had quicker latency scores than old-old adults regardless of whether they were engaging in regular physical activity or sedentary. The results also showed that young-old physically active adults scored better on the latency measure than sedentary adults however the opposite was found for old-old adults; physically active oldold adults scored worse than sedentary adults on the latency measure. This result goes against previous research that found that performance on digit span tasks to be better with physically active older adults than sedentary older adults [31].

When exploring the DST the results found that young-old adults were able to recall larger digit spans and had quicker latency scores than old-old adults regardless of whether they were engaging in regular physical activity or sedentary. This result was expected as younger adults have been shown to have better cognitive functioning compared to older adults [19]. The results of the current study also showed that young-old physically active adults scored better on the latency measure than sedentary adults however the opposite was found for old-old adults; physically active old-old adults scored worse than sedentary adults on the latency measure. This result goes against previous research that found that performance on digit span tasks to be better with physically active older adults than sedentary older adults [31]. Oldold sedentary adults scoring better than physically 
active old-old participants on the latency measure of the DST could be explained through research showing a relationship between acute exercise and arterial stiffness, whereby blood takes longer to travel to different brain areas [49, 50]. Some of these participants could be engaging in acute exercise therefore possibly causing an inflammatory response and effecting cognitive functioning $[49,50]$.

The results for the WCST showed that there was no significant main effect among age and physical activity. The lack of significant differences in cognitive functioning using the WCST could be because of boredom effects. It was noticeable when participants were completing the WCST that they became frustrated with the task and this could have impacted the results. Physically active were those who engaged in aerobic or muscle-strengthening activity that was planned, structured and required repetitive movement that allowed for improved or maintained level of physical fitness. The loose criteria of what could be considered physical activity could have had an effect on the findings. Despite not finding any significant differences with the WCST previous studies having more control over the physical activity variable have found significant differences in older adults [51]. Future research should therefore continue to use these measures when exploring the relationship between cognitive functioning and physical activity. Based on these results the first hypothesis was partially supported.

The NTG-EDSD is a fairly new measure designed as an early screening tool for dementia [25]. There was no established or normed scoring system for this measure, consequently, a scoring system for this measure had to be adopted in the current study. The results of the NTG-EDSD indicated that physically active adults (young-old and old-old) scored significantly lower than sedentary adults (young-old and old-old) therefore suggesting that based on self-reports older adults that engage in physical activity have better thinking, behavior and adaptive skills [15]. The current study was the first to explore the relationship between the NTG-EDSD and older adults. Based on the results of the NTG-EDSD the second hypothesis was supported.

The findings of this research provide insights into the effects of cognitive functioning in older adults. Specifically, extending our knowledge of the differences between physical activity and cognitive functioning. The studies limitations also provide further implications for future research in that future research should have more control over the independent variables to further understand the relationships between measures. Future research could also focus on the development of certain measures for detecting early signs of cognitive decline needs to help deal with the dementia and create in order to reserve cognitive functioning. Although new measures like the NTG-EDSD are being established, proper procedures and scoring need to be established in order to make more accurate conclusions about the diagnosis. Future research should also diversify the physical activity group based on specific activities rather than include all types into one group.

\section{CONFLICT OF INTEREST}

The authors have no conflict of interest to report.

\section{REFERENCES}

[1] Australian Institute of Health and Welfare (2012). Dementia in Australia (cat No. 70). AIHW, Canberra, ACT. Retrieved from http://www.aihw.gov.au/publicationdetail/?id=10737422958

[2] Deary IJ, Corley J, Gow AJ, Harris SE, Houlihan LM, Marioni RE, Penke L, Rafnsson SB, Starr JM (2009) Ageassociated cognitive decline. Br Med Bull 92, 135-152.

[3] O'Sullivan M1, Jones DK, Summers PE, Morris RG, Williams SC, Markus HS (2001) Evidence for cortical "disconnection" as a mechanism of age-related cognitive decline. Neurology 57, 632-638.

[4] Fillit H (1997) The clinical significance of normal cognitive decline in later life. In Cognitive decline: Strategies for Prevention, Fillit HM, Butler RN, eds. University Press, Cambridge, UK.

[5] Australian Institute of Health and Welfare (2012) Dementia in Australia (cat No. 70). AIHW, Canberra, ACT. Retrieved from http://www.aihw.gov.au/publicationdetail/?id=10737422958

[6] World Health Organization, \& International Alzheimer's Disease (2012) Dementia a public health priority. World Health Organization 112. Retrieved from http://www.who. int/mental_health/publications/dementia_report_2012/en/

[7] Fritsch T, McClendon MJ, Smyth KA, Lerner AJ, Friedland RP, Larsen JD (2007) Cognitive functioning in healthy aging: The role of reserve and lifestyle factors early in life. Gerontologist 47, 307-322.

[8] Stint-Morrow EAL, Chui H (2012) Cognitive resilience in adulthood. Ann Rev Gerontol Geriatr 32, 93-114.

[9] Colcombe SJ, Erickson KI, Scalf PE, Kim JS, Prakash R, McAuley E, Elavsky S, Marquez DX, Hu L, Kramer AF (2006) Aerobic exercise training increases brain volume in aging humans. J Gerontol A Biol Sci Med Sci 61, 1166-1170.

[10] Erickson KI, Raji CA, Lopez OL, Becker JT, Rosano C, Newman AB, Gach HM, Thompson PM, Ho AJ, Kuller LH (2010) Physical activity predicts grey matter volume in late adulthood: The Cardiovascular Health Study. Neurology 75, 1415-1422.

[11] Smith JC, Nielson KA, Woodard JL, Seidenberg M, Rao SM (2013) Physical activity and brain function in older adults at increased risk for Alzheimer's disease. Brain Sci 3, 54-83. 
[12] Ahlskog JE, Geda YE, Graff-Radford NR, Petersen RC (2011) Physical exercise as a preventive or diseasemodifying treatment of dementia and brain aging. Mayo Clinic Proc 86, 876-884.

[13] Yu F, Nelson NW, Savik K, Wyman JF, Dysken M, Bronas UG (2013) Affecting cognition and quality of life via aerobic exercise in Alzheimer's disease. West J Nurs Res 35, 24-38.

[14] Pittenger C, Duman RS (2008) Stress, depression, and neuroplasticity: A convergence of mechanisms. Neuropsychopharmacology 33, 88-109.

[15] Shaffer J (2012) Neuroplasticity and positive psychology in clinical practice: A review for combined benefits. Psychology 3, 1110-1115.

[16] Vance DE, Roberson AJ, McGuinness TM, Fazeli PL (2010) How neuroplasticity and cognitive reserve protect cognitive functioning. J Psychosoc Nurs Ment Health Serv 48, 23-30.

[17] Scarmeas N, Stern Y (2003) Cognitive reserve and lifestyle. J Clin Exp Neuropsychol 25, 625-633.

[18] Cotman CW, Berchtold NC (2002) Exercise: A behavioural intervention to enhance brain health and plasticity. Trends Neurosci 25, 295-301.

[19] Colcombe S, Kramer AF (2003) Fitness effects on the cognitive function of older adults: A meta-analytic study. Psychol Sci 14, 125-130.

[20] Newson RS, Kemps EB (2006) The influence of physical and cognitive activities on simple and complex cognitive tasks in older adults. Exp Aging Res 32, 341-362.

[21] Small BJ, Dixon RA, Hultsch DF, Hertzog C (1999) Longitudinal changes in quantitative and qualitative indicators of word and story recall in young-old and old-old adults. J Gerontol 54B, 107-115.

[22] Ashford JW (2010) Screening tools for MCI. Alzheimers Dement 6, S91-S91.

[23] Hildebrandt H, Haldenwanger A, Eling P (2009) False recognition helps to distinguish patients with Alzheimer's disease and amnestic MCI from patients with other kinds of dementia. Dement Geriatr Cogn Disord 28, 159-167.

[24] Hoops S, Nazem S, Siderowf AD, Duda JE, Xie SX, Stern MB, Weintraub D (2009) Validity of the MoCA and MMSE in the detection of MCI and dementia in Parkinson disease. Neurology 73, 1738-1745.

[25] Esralew L, Janicki MP, Jokinen M, Keller SM (2013) National task group early detection screen for dementia: Manual. Retrieved from http://aadmd.org/sites/default/ files/NTGEDSDmanual-bangor version-e.pdf

[26] Butler M, Retzlaff P, Vanderploeg R (1991) Neuropsychological test usage. Prof Psychol Res Pr 22, 510-512.

[27] Strauss E, Sherman EMS, Spreen O (2006) A compendium of Neuropsychological tests: Administration, norms, and commentary, 3rd ed. Oxford University Press, NY.

[28] Steinmetz JP, Brunner M, Loarer E, Houssemand C (2010) Incomplete psychometric equivalence of scores obtained on the manual and the computer version of the Wisconsin Card Sorting Test? Psychol Assess 22, 199-202.

[29] Bopp KL, Verhaeghen P (2005) Aging and verbal memory span: A-meta analysis. J Gerontol 60, 223-233.

[30] Feldman HH, Jacova C, Robillard A, Garcia A, Chow T, Borrie M, Schipper HM, Blair M, Kertesz A, Chertkow H (2008) Diagnosis and treatment of dementia: 2. Diagnosis. CMAJ 178, 825-836.

[31] Kemtes KA, Allen DN (2008) Presentation modality influences WAIS Digit Span performance in younger and older adults. J Clin Exp Neuropsychol 30, 661-665.
[32] Lezak MD, Howieson DB, Loring DW (2004) Neuropsychological assessment, 4th ed. Oxford University Press, NY.

[33] Myerson J, Emery L, White DA, Hale S (2003) Effects of age, domain, and processing demands on memory span: Evidence for differential decline. Aging Neuropsychol Cogn 10, 20-27.

[34] Olsthoorn NM, Andringa SJ, Hulstijn JH (2014) Visual and auditory digit-span performance in native and nonnative speakers. Int J Billing 18, 663-673.

[35] Taub HA (1972) A comparison of young adult and old groups on various digit span tasks. Dev Psychol 6, 60-65.

[36] Cassilhas RC, Viana VA, Grassmann V, Santos RT, Santos RF, Tufik S, Mello MT (2007) The impact of resistance exercise on the cognitive function of the elderly. Med Sci Sports Exerc 39, 1401-1407.

[37] Dustman RE, Ruhling RO, Russell EM, Shearer DE, Bonekat HW, Shigeoka JW, Wood JS, Bradford DC (1984) Aerobic exercise training and improved neuropsychological function of older individuals. Neurobiol Aging 5, 35-42.

[38] Nelson ME, Rejeski WJ, Blair SN, Duncan PW, Judge JO, King AC, Macera CA, Castaneda-Sceppa C, American College of Sports Medicine, American Heart Association (2007) Physical activity and public health in older adults: Recommendation from the American College of Sports Medicine and the American Heart Association. Circulation 116, 1094-1105.

[39] Hester RL, Kinsella GJ, Ong B (2004) Effect of age on forward and backward span tasks. J Int Neuropsychol Soc 10, 475-481.

[40] GrÉGoire J, Van Der Linden M (1997) Effect of age on forward and backward digit spans. Aging Neuropsychol Cogn 4, 140-149.

[41] Mueller ST (2011) The PEBL digit span test [Digit span test]. Retrieved from http://pebl.sf.net

[42] Woods DL, Kishiyamaa MM, Lund EW, Herron TJ, Edwards B, Poliva O, Hink RF, Reed B (2011) Improving digit span assessment of short-term verbal memory. J Clin Exp Neuropsychol 33, 101-111.

[43] Rhodes MG (2004) Age-related differences in performance on the Wisconsin card-sorting test: A meta-analytic review. Psychol Aging 19, 482-494.

[44] Lovibond SH, Lovibond PF (1995) Manual for the Depression Anxiety Stress Scales, 2nd ed. Psychology Foundation, Sydney.

[45] Antony MM, Bieling PJ, Cox BJ, Enns MW, Swinson RP (1998) Psychometric properties of the 42-Item and 21-Item versions of the depression anxiety stress scales in clinical groups and a community sample. Psychol Assess 10, 176181.

[46] Osman A, Wong JL, Bagge CL, Freedenthal S, Gutierrez PM, Lozano G (2012) The Depression Anxiety Stress Scales-21 (DASS-21): Further examination of dimensions, scale reliability, and correlates. J Clin Psychol 68, 13221338.

[47] Henry JD, Crawford JR (2005) The short-form version of the Depression Anxiety Stress Scales (DASS-21): Construct validity and normative data in a large non-clinical sample. Br J Clin Psychol 44, 227-239.

[48] Brown TA, Chorpita BF, Korotitsch W, Barlow DH (1997) Psychometric properties of the Depression Anxiety Stress Scales (DASS) in clinical samples. Behav Res Ther 35, 79-89.

[49] Kokkinos P (2012) Physical activity, health benefits, and mortality risk. ISRN Cardiol 2012, 718789. 
[50] Lee HY, Oh BH (2010) Aging and arterial stiffness. Circ J 74, 2257-2262.

[51] Voss MW, Heo S, Prakash RS, Erickson KI, Alves H, Chaddock L, Szabo AN, Mailey EL, Wójcicki TR, White SM, Gothe N, McAuley E, Sutton BP, Kramer AF (2013) The influence of aerobic fitness on cerebral white matter integrity and cognitive function in older adults: Results of a one-year exercise intervention. Hum Brain Mapp 34, 2972-2985. 